\title{
SURFACES IN A CONFORMAL CORRESPONDENCE
}

\author{
T. K. PAN
}

TO THE MEMORY OF THE AUTHOR'S WIFE, HELEN WU PAN

1. Introduction. Two surfaces $S$ and $\bar{S}$ are said to be in conformal correspondence if there exists a one-to-one correspondence between their points such that the angle between two directed curves through a point $P$ of $S$ is equal always to the angle between the two corresponding directed curves through the corresponding point $\bar{P}$ of $\bar{S}$. A necessary and sufficient condition that $S$ and $\bar{S}$ be in conformal correspondence is that between their linear elements $d s^{2}$ and $d \bar{s}^{2}$ there exists a relation of the form $d \bar{s}^{2}=u^{2} d s^{2}$. When $u$ is constant, $S$ and $\bar{S}$ are said to be similar.

Let $v$ and $\bar{v}$ be two unit vector fields associated to $S$ and $\bar{S}$, respectively. A curve on $S$ along which the vectors of $v$ are tangent to the curve, is called the curve of $v$ on $S$. A curve on $S$ relative to which the vectors of $v$ are parallel in the sense of Levi-Civita, is called the indicatrix of $v$ on $S$. Along a curve $C$ on $S$, the derived vector of $v$ can be decomposed into a component tangential and a component normal to $S$. The magnitude of the normal component is called the normal curvature of $v$ with respect to $C$. In a conformal correspondence between $S$ and $\bar{S}$, suppose that: (1) the curve of $v$ on $S$ corresponds to the curve of $\bar{v}$ on $\bar{S},(2)$ the indicatrix of $v$ on $S$ corresponds to the indicatrix of $\bar{v}$ on $\bar{S}$, and (3) the normal curvature of $v$ with respect to any curve $C$ on $S$ is equal to the normal curvature of $\bar{v}$ with respect to the corresponding curve $\bar{C}$ on $\bar{S}$. This paper intends to study the existence of these surfaces and their degree of generality.

In order to facilitate the investigation, exterior differential calculus and the method of moving trihedrals are employed. The notation of Cartan [1] will be used for the most part except that $\wedge$ will be adopted as the symbol of exterior multiplication.

2. Differential system in involution. Any system of differential equations may be reduced to a Pfaffian system, by which we mean a differential system consisting of equations of the form

$$
\begin{array}{rr}
F_{\alpha} \equiv f_{\alpha}\left(x^{1}, \cdots, x^{n}\right)=0, & \alpha=1, \cdots, r_{0}, \\
\theta_{\beta} \equiv A_{\beta i}\left(x^{1}, \cdots, x^{n}\right) d x^{i}=0, & \beta=1, \cdots, r_{1}, i=1, \cdots, n,
\end{array}
$$

Presented to the Society, August 24, 1956, under the title Exterior differential system and mapping of surfaces with associated vector fields in correspondence; received by the editors August 8, 1956. 
where the first set of equations may possibly be absent. ${ }^{1}$ The system (2.1) is said to be closed if we adjoin to it the following equations

$$
\begin{aligned}
d F_{\alpha} & \equiv \frac{\partial f_{\alpha}}{\partial x^{i}} d x^{i}=0, \\
d \theta_{\beta} & \equiv d A_{\beta i} \wedge d x^{i}=0,
\end{aligned}
$$

which are obtained by equating to zero the exterior differentials of the left-hand members of equations (2.1).

Suppose a closed differential system $\Sigma$ consists of the following equations

$$
\begin{aligned}
F_{\alpha} \equiv f_{\alpha}\left(x^{1}, \cdots, x^{n}\right)=0, & \alpha=1, \cdots, r_{0}, \\
\theta_{\beta} \equiv A_{\beta i}\left(x^{1}, \cdots, x^{n}\right) d x^{i}=0, & \beta=1, \cdots, r_{1}, \\
\psi_{\gamma} \equiv \frac{1}{2} A_{\gamma i j}\left(x^{1}, \ldots, x^{n}\right) d x^{i} \wedge d x^{j}=0, & \gamma=1, \cdots, r_{2}, \\
. . . . . . . . . . . . . & i, j=1, \cdots, n
\end{aligned}
$$

which are of degrees $0,1,2, \cdots$, respectively.

Let $E_{p}$ be a contact element of dimension $p$ in an arbitrary $n$ dimensional manifold $V$, which is meant a pair $(M, \pi)$ formed by a point $M$ of $V$ and a $p$-dimensional vector subspace $\pi$ of the $n$ dimensional tangent vector space to $V$ at $M$. A contact element $E_{p}$ is called an integral element of dimension $p$ of the closed differential system $\Sigma$, if its origin $M$ is a point of the analytic manifold $V_{0}$ defined by the finite equations $F_{\alpha}=0$ of $\Sigma$, and if any $h$ vectors of $\pi$ from $M$ satisfy all equations of degree $h$ in $\Sigma$ at $M$ for $h=1, \cdots, p$. Let $W$ be a $p$-dimensional submanifold of $V$. If every contact element $E_{p}$, which is tangent to $W$ at its origin, is an integral element of $\Sigma$, then $W$ is called a $p$-dimensional solution or integral manifold of $\Sigma$. Any solution of a differential system is a solution of its closed system.

Let $r$ be the dimension of $V_{0}$. A point of $V_{0}$ is called a simple point, if at the point the matrix $\left\|\partial f_{\alpha} / \partial x^{i}\right\|$ is of rank $n-r$. Denote by $s_{0}$ the rank of the matrix $\left\|A_{\beta i}\right\|$ at a generic simple point. ${ }^{2}$ If, at a point, all one-dimensional integral elements of $\Sigma$ span a contact element with origin at the point and with a dimension not greater than $n-s_{0}$, then this point is called an ordinary point. An integral element

1 We agree as usual that repeated indices mean summation.

2 The linear equations of $\Sigma$ at the generic simple point may be said to form the polar system of the point. If a differential system does not consist of finite equations, we define $s_{0}=r_{1}$, the number of linearly independent equations of degree 1 in the system $[1$, p. 98]. 
of dimension 1 with an ordinary point as its origin is called regular. Let $E_{1}$ be a regular integral element of dimension 1 with components $u^{i}$. The locus of contact elements $d x^{i}$ at the origin of $E_{1}$, such that $u^{i}$ and $d x^{i}$ determine an integral element of dimension 2 of $\Sigma$, is defined by

$$
\begin{aligned}
A_{\beta i} d x^{i} & =0, \\
A_{\gamma i j} u^{i} d x^{j} & =0,
\end{aligned}
$$

with $A_{\beta i} u^{i}=0$. The system of equations (2.4) is called the polar system of $E_{1}$. Denote by $s_{0}+s_{1}$ the rank of the matrix of (2.4), when $E_{1}$ is generic. A regular integral element $E_{1}$ is called ordinary if the matrix of its polar system is of rank not less than $s_{0}+s_{1}$. An integral element of dimension 2 is called regular if it contains at least one ordinary integral element of dimension 1 . Continuing in the same manner, we may define ordinary and regular integral elements of dimension $p$ and the number $s_{0}+s_{1}+\cdots+s_{p-1}$. When $s_{0}+s_{1}+\cdots$ $+s_{h}=n-h$, there does not exist a regular integral element of dimension $h+1$. The number $h$ is called the genus of $\Sigma$; the numbers $s_{0}, s_{1}, \cdots, s_{h}$ are called the characters of orders $0,1, \cdots, h$ of $\Sigma$, respectively.

When a closed differential system $\Sigma$ is expressed in $n-p$ unknown functions of $p$ independent variables $x^{1}, x^{2}, \cdots, x^{p}$, the system is said to be in involution if its genus is not less than $p$ and if the equations of its generic regular integral elements of dimension $p$ do not introduce linear relation between $d x^{1}, d x^{2}, \cdots, d x^{p}$. The integral manifold of $\Sigma$, which is of dimension $p$ with independent parameters $x^{1}, x^{2}, \cdots, x^{p}$, may be obtained by applying the following existence theorem [1, p. 68].

EXISTENCE THEOREM. Let $E_{p}$ be a regular integral element of dimension $p$ and $V_{p-1}$ an integral manifold of dimension $p-1$ tangent to an ordinary integral element $E_{p-1}$ contained in $E_{p}$. If $s_{0}+s_{1}+\cdots+s_{p-1}$ $\leqq n-p$, there exists at least one integral manifold of dimension $p$, which contains $V_{p-1}$ and is tangent to $E_{p}$.

There are several ways to distinguish systems in involution from systems not in involution. In particular, when $p=2$, that is, when there are only two independent variables, we shall repeatedly apply the following theorem [1, p. 100].

Sufficient condition of involution. A closed differential system in two independent variables is in involution if its reduced character of order 1 is equal to the number of linearly independent quadratic forms in the system. 
The reduced characters of orders 0 and 1 , denoted by $s_{0}^{\prime}$ and $s_{1}^{\prime}$, are the characters $s_{0}$ and $s_{1}$, when the differentials of the independent variables are disregarded in the polar systems. We define $s_{2}^{\prime}$ by the relation $s_{0}^{\prime}+s_{1}^{\prime}+s_{2}^{\prime}=n-2$.

With regard to exterior differential system, only those definitions and theorems used in this investigation are stated in the above. An account in detail may be found in [1] and [3], an excellent brief exposition appears in [2].

3. General solution. Let $F$ be a family of moving rectangular trihedrals, $A e_{1} e_{2} e_{3}$, with $A$ as origin. An infinitesimal displacement of $F$ is defined by

$$
\begin{aligned}
d A & =\omega^{i} e_{i}, \\
d e_{i} & =\omega_{i}^{i} e_{j},
\end{aligned}
$$

where $\omega^{i}, \omega_{i}^{j}=\omega_{i j}$ are linear differential forms called the relative components of the displacement. Since $\omega_{1}^{1}=\omega_{2}^{2}=\omega_{3}^{3}=0$ and $\omega_{i}^{j}=-\omega_{j}^{i}$, there are essentially only six such forms.

The relative components satisfy the following equations of structure

$$
\begin{aligned}
d \omega^{i} & =\omega^{k} \wedge \omega_{k}^{i}, \\
d \omega_{i}^{j} & =\omega_{i}^{k} \wedge \omega_{k}^{j} .
\end{aligned}
$$

Conversely, any six linear differential forms $\omega^{i}, \omega_{i}^{j}=-\omega_{j}^{j}$ in the variables $u^{q}$ and their differentials $d u^{q}, q \leqq 6$, which satisfy (3.2), are relative components of an infinitesimal displacement of a family of rectangular trihedrals. Hence the research of a problem in differential geometry may be reduced to the research of a family of trihedrals attached.

Take $e_{3}$ to be the surface normal and $e_{1}$ the associated vector on both $S$ and $\bar{S}$. Then the curves of $v$ and $\bar{v}$ are defined by $\omega^{2}=0$ and $\bar{\omega}^{2}=0$, respectively. From [4, pp. 956-957] and

$$
d e_{1}=\stackrel{2}{\omega_{1} e_{2}}+\stackrel{3}{\omega_{1} e_{3}},
$$

it is obvious that the indicatrices of $v$ and $\bar{v}$ are defined respectively by $\omega_{12}=0$ and $\bar{\omega}_{12}=0$, and that $\omega_{1}^{3} / d s$ and $\bar{\omega}_{1}^{3} / d \bar{s}$ are respectively the normal curvatures of $v$ and $\bar{v}$, where $d s$ and $d \bar{s}$ are the arc lengths of the corresponding curves along which $e_{1}$ is displaced.

Our proposed problem, that is, to find surfaces $S$ and $\bar{S}$ in a conformal correspondence with properties (1), (2), (3) as described in the Introduction, is then given by the following differential system 


$$
\begin{aligned}
\omega^{3} & =0, & \bar{\omega}^{3} & =0, \\
\bar{\omega}_{1} & =u \omega^{1}, & \bar{\omega}^{2} & =u \omega^{2}, \\
\bar{\omega}_{13} & =u \omega_{13}, & \bar{\omega}_{12} & =v \omega_{12} .
\end{aligned}
$$

Exterior differentiation of (3.3) gives

$$
\begin{array}{r}
\omega^{1} \wedge \omega_{13}+\omega^{2} \wedge \omega_{23}=0, \\
u \omega^{1} \wedge \omega_{13}+\omega^{2} \wedge \bar{\omega}_{23}=0, \\
\omega^{1} \wedge \frac{d u}{u}+(1-v) \omega^{2} \wedge \omega_{12}=0, \\
\omega^{2} \wedge \frac{d u}{u}-(1-v) \omega^{1} \wedge \omega_{12}=0, \\
\omega_{13} \wedge \frac{d u}{u}-\omega_{12} \wedge \omega_{23}+\frac{v}{u} \omega_{12} \wedge \bar{\omega}_{23}=0, \\
\omega_{12} \wedge \frac{d v}{v}+\omega_{13} \wedge \omega_{23}-\frac{u}{v} \omega_{13} \wedge \bar{\omega}_{23}=0 .
\end{array}
$$

The generic integral element of dimension 2 is given by the equations

$$
\begin{aligned}
& \omega_{13}=a \omega^{1}+b \omega^{2}, \\
& \omega_{23}=b \omega^{1}+c \omega^{2}, \\
& \omega_{12}=h \omega^{1}+k \omega^{2}, \\
& \bar{\omega}_{23}=\alpha \omega^{1}+\beta \omega^{2}, \\
& \frac{d u}{u}=\gamma \omega^{1}+\delta \omega^{2}, \\
& \frac{d v}{v}=\lambda \omega^{1}+\mu \omega^{2},
\end{aligned}
$$

in which the coefficients satisfy the following five relations

$$
\begin{gathered}
\alpha=u b, \quad \delta=h(1-v), \quad \gamma=k(v-1), \\
a \delta-b \gamma+b k-c h+\frac{v}{u}(h \beta-\alpha k)=0, \\
h \mu-k \lambda+a c-b^{2}-\frac{u}{v}(a \beta-b \alpha)=0 .
\end{gathered}
$$

The matrix of the reduced polar system of (3.4) has for its determinant with columns relative to the forms $\omega_{13}, \omega_{23}, \omega_{12}, \bar{\omega}_{23}, d u / u, d v / v$ 


$$
\begin{array}{|cccccc}
\omega^{1} & \omega^{2} & 0 & 0 & 0 & 0 \\
u \omega^{1} & 0 & 0 & \omega^{2} & 0 & 0 \\
0 & 0 & (1-v) \omega^{2} & 0 & \omega^{1} & 0 \\
0 & 0 & -(1-v) \omega^{1} & 0 & \omega^{2} & 0 \\
-\frac{d u}{u} & -\omega_{12} & \omega_{23}-\frac{v}{u} \bar{\omega}_{23} & \frac{v}{u} \omega_{12} & \omega_{13} & 0 \\
-\omega_{23}+\frac{u}{v} \bar{\omega}_{23} & \omega_{13} & -\frac{d v}{v} & -\frac{u}{v} \omega_{13} & 0 & \omega_{12}
\end{array} \mid
$$

the right-hand expression being obtained by help of (3.5). Thus, $s_{1}^{\prime}=6$, the number of equations in (3.4). It is easy to find that $s_{2}^{\prime}=0$. Hence, by the two theorems stated in $\$ 2$, we conclude that the closed system (3.3), (3.4) is in involution and its general solution depends on six arbitrary functions of one variable. Consequently we have

THEOREM 3.1. There exist surfaces in conformal correspondence preserving the curves, indicatrices and normal curvatures of associated unit vector fields. The general solution depends on six arbitrary functions of one variable. The characteristics are the curves and the indicatrices of the associated vector fields.

The characteristics are curves which are contained in the general solution and whose tangent elements are not ordinary. The last part of the above theorem is obvious from (3.7).

4. Singular solution. A singular solution is a solution not contained in the general solution. The singular solutions of any closed system, if they exist, may be obtained by a method of prolongation [1, pp. 112-120]. For the closed system (3.3) and (3.4), it is to adjoin $v-1=0$ to the system and solve the resulting new system.

When $v=1$, equations (3.4) give $d u=0$. The closed system which gives the singular solutions is defined by

$$
\begin{gathered}
\omega^{3}=0, \quad \bar{\omega}^{3}=0, \quad \bar{\omega}_{12}=\omega_{12}, \\
\bar{\omega}^{1}=m \omega^{1}, \quad \bar{\omega}^{2}=m \omega^{2}, \quad \bar{\omega}_{13}=m \omega_{13}, \\
\omega^{1} \wedge \omega_{13}+\omega^{2} \wedge \omega_{23}=0, \\
m \omega^{1} \wedge \omega_{13}+\omega^{2} \wedge \bar{\omega}_{23}=0, \\
\omega_{12} \wedge \bar{\omega}_{23}-m \omega_{12} \wedge \omega_{23}=0, \\
m_{13} \wedge \bar{\omega}_{23}-\omega_{13} \wedge \omega_{23}=0,
\end{gathered}
$$

$m$ being a constant different from zero. 
The determinant of the polar matrix with columns relative to the forms $\omega_{13}, \omega_{23}, \bar{\omega}_{23}, \omega_{12}$ is as follows

$$
\begin{aligned}
& \left|\begin{array}{cccc}
\omega^{1} & \omega^{2} & 0 & 0 \\
m \omega^{1} & 0 & \omega^{2} & 0 \\
0 & -m \omega_{12} & \omega_{12} & m \omega_{23}-\bar{\omega}_{23} \\
m \bar{\omega}_{23}-\omega_{23} & \omega_{13} & -m \omega_{13} & 0
\end{array}\right| \\
& =\left(\bar{\omega}_{23}-m \omega_{23}\right)\left[\left(\omega^{2}\right)^{2}\left(m \bar{\omega}_{23}-\omega_{23}\right)+\omega_{13} \omega^{1}\left(m^{2} \omega^{2}-\omega_{12}\right)\right] .
\end{aligned}
$$

Since $s_{0}^{\prime}=6, s_{1}^{\prime}=4, n=12, s_{2}^{\prime}=0$, the system (4.1) is in involution and its general solution depends on four arbitrary functions of one variable.

The generic integral element of dimension 2 is given by

$$
\begin{aligned}
& \omega_{13}=a \omega^{1}+b \omega^{2}, \\
& \omega_{23}=b \omega^{1}+c \omega^{2}, \\
& \bar{\omega}_{23}=m b \omega^{1}+\beta \omega^{2}, \\
& \omega_{12}=h \omega^{1}+k \omega^{2},
\end{aligned}
$$

with the following relations

$$
\begin{aligned}
h(\beta-m c) & =0, \\
a c-b^{2}-m\left(a \beta-m b^{2}\right) & =0 .
\end{aligned}
$$

We have from (4.3) either

$$
h=0, \quad a c-b^{2}-m\left(a \beta-m b^{2}\right)=0,
$$

which imply

$$
\omega_{12}=k \omega^{2}, \quad \omega_{13} \wedge \omega_{23}=\bar{\omega}_{13} \wedge \bar{\omega}_{23}
$$

or

$$
\beta-m c=0, \quad a c-b^{2}-m\left(a \beta-m b^{2}\right)=0,
$$

which imply

$$
m= \pm 1 \text { or } \omega_{13} \wedge \omega_{23}=\bar{\omega}_{13} \wedge \bar{\omega}_{23}=0 .
$$

Hence we obtain

THEOREM 4.1. The singular solution depends on four arbitrary functions of one variable. It consists of: equivalent or symmetrical surfaces associated with equivalent unit vector fields; similar developable surfaces with associated unit vector fields whose angular spreads with respect to 
corresponding curves on the surfaces are inversely proportional to their arc lengths; similar surfaces with equal Gaussian curvatures at corresponding points and with associated unit vector fields whose curves are geodesics and whose angular spreads with respect to corresponding curves are inversely proportional to their arc lengths.

5. Cauchy problem. The problem of determining an integral manifold of dimension $p$ containing a given manifold of dimension $p-1$ has been called the Cauchy problem. We proceed to discuss it relative to the general solution.

Take two arbitrary curves $C$ and $\bar{C}$, which are considered to be corresponding curves. Associate a unit vector at each point of these curves, denoted by $v$ on $C$ and by $\bar{v}$ on $\bar{C}$. Attach at each point of $C$ a direct rectangular trihedral such that the vector $e_{1}$ coincides with the vector $v$ and that the vector $e_{3}$ is normal to $C$. Let $\theta$ be the angle which $C$ makes with $e_{1}$. Let $\omega$ be the angle which the principal normal of $C$ makes with $e_{3}$. Let $1 / \rho$ and $1 / \tau$ be the absolute curvature and torsion of $C$, respectively. We attach similarly a direct rectangular trihedral to $\bar{C}$ and define in the same manner $\bar{\theta}, \bar{\omega}, 1 / \bar{\rho}$, and $1 / \bar{\tau}$ for $\bar{C}$. Then the curves $C$ and $\bar{C}$ constitute a one-dimensional integral manifold of the system (3.3), if we have

$$
\begin{aligned}
d \bar{s} & =u d s, \\
u\left(\frac{\sin \bar{\omega}}{\bar{\rho}}-\frac{d \bar{\theta}}{d \bar{s}}\right) & =v\left(\frac{\sin \omega}{\rho}-\frac{d \theta}{d s}\right),
\end{aligned}
$$

$$
\cos \bar{\theta} \frac{\cos \bar{\omega}}{\bar{\rho}}-\sin \bar{\theta}\left(\frac{d \bar{\omega}}{d \bar{s}}+\frac{1}{\bar{\tau}}\right)=u \cos \theta \frac{\cos \omega}{\rho}-u \sin \theta\left(\frac{d \omega}{d s}+\frac{1}{\tau}\right) .
$$

The last two equations in (5.1) are obtained by use of the following relations

$$
\begin{gathered}
\frac{1}{R_{n}}=\frac{\cos \omega}{\rho}, \quad \frac{d s}{R_{g}}=d \theta+\omega_{12}, \quad \frac{d s}{T_{g}}=d \omega+\frac{d s}{\tau}, \\
\omega_{1} \mathrm{II}-\omega_{2} \mathrm{III}=\omega_{13} \mathrm{I},
\end{gathered}
$$

where $1 / R_{n}, 1 / R_{\theta}$, and $1 / T_{\theta}$ are respectively the normal curvature, geodesic curvature and geodesic torsion of $C$, and where I, II, and III denote respectively the first, second, and third fundamental forms of $S$.

By assumption, we know $\theta$ and $\bar{\theta}$ as functions of $s$ and $\bar{s}$, respectively, $\theta$ being equal to $\bar{\theta}$ at corresponding points. Also we know $1 / \rho, 1 / \tau$ and $\omega$ as functions of $s ; 1 / \bar{\rho}, 1 / \bar{\tau}$ and $\bar{\omega}$ as functions of $\bar{s}$. 
Hence $v$ and $u$ can be determined by the last two equations of (5.1). The first equation of (5.1) then gives the relation between $s$ and $\bar{s}$. Thus, by the existence theorem, we obtain uniquely two surfaces $S$ and $\bar{S}$, which contain the corresponding curves $C$ and $\bar{C}$, which have their associated vector fields containing $v$ and $\bar{v}$ along $C$ and $\bar{C}$, and which are in conformal correspondence preserving the curves, indicatrices and normal curvatures of the associated vector fields. Given $\theta$, which is equal to $\bar{\theta}$, the corresponding curves $C$ and $\bar{C}$ depend on six functions of one variable, namely, $1 / \rho, 1 / \tau, \omega, 1 / \bar{\rho}, 1 / \bar{\tau}$, and $\bar{\omega}$. Therefore Theorem 3.1 is verified once again.

If $\theta=\bar{\theta}=0, C$ and $\bar{C}$ are the curves of the associated vector fields. Then there are infinitely many ways of taking $e_{3}$ normal to $C$ or to $\bar{C}$. Consequently, $\omega$ and $\bar{\omega}$ are not unique and the Cauchy problem has infinitely many solutions. If $C$ and $\bar{C}$ are the indicatrices of the associated vector fields, then the second equation of (5.1) is satisfied by any value of $v$. Hence the Cauchy problem has also infinitely many solutions. Both the curves and the indicatrices of the associated vector fields are the characteristics as stated in Theorem 3.1.

\section{REFERENCES}

1. E. Cartan, Les systèmes différentials extérieurs et leurs applications géométriques, Paris, Hermann, 1945.

2. S. S. Chern and C. Chevalley, Elie Cartan and his mathematical work, Bull. Amer. Math. Soc. vol. 58 (1952) pp. 217-250.

3. E. Kähler, Einführung in die Theorie der Systeme von Differentialgleichungen, Hamburger Mathematische Einzelschriften vol. 16 (1934).

4. T. K. Pan, Normal curvature of a vector field, Amer. J. Math. vol. 74 (1952) pp. $955-966$.

UNIVERSITY OF OKLAHOMA 